\title{
Innate lymphoid cells and type 2 (Th2) mediated immune responses - pathogenic or beneficial?
}

\section{Christoph Wilhelm and Brigitta Stockinger*}

Division of Molecular Immunology, MRC National Institute for Medical Research, London, UK

*Correspondence: bstocki@nimr.mrc.ac.uk

The last 5 years have seen a remarkable extension of the innate immune system starting with what seemed at the time primarily cells that exert their influence during ontogeny of the immune system. So-called lymphoid tissue inducer (Lti) cells were soon joined by RORgt expressing innate lymphoid cells (ILC) that shaped immune responses beyond the determination of lymphoid architecture (Sawa et al., 2010). The latest addition to the growing family of ILC are characterized by the production of T helper (Th) 2-type cytokines even in the absence of the adaptive immune system (Moro et al., 2010; Neill et al., 2010; Price et al., 2010; Saenz et al., 2010).

$\mathrm{T}$ helper 2-type cytokine responses play a major role in the immunity to parasitic helminth infections, but are also implicated in the development of allergic diseases such as asthma (Paul and Zhu, 2010). The hallmark of Th2 mediated immune responses is the activation of mast cells, eosinophils, basophils, and goblet cell hyperplasia mediated by the cytokines IL-5, IL-9, and IL-13. Until recently, $\mathrm{T}$ cells of the Th cell type 2 (Th2) subset were thought to be the most abundant and important source of these cytokines. However, it became clear that a group of innate cells exist, that lack conventional markers typical for main lymphoid cell types and are able to produce high amounts of Th2-type cytokines (Fort et al., 2001; Hurst et al., 2002; Fallon et al., 2006). Such responses were shown to be dependent on the epithelial cell derived cytokine IL-25. More recently those ILC populations were further characterized by four independent research groups and called natural helper cells (NHCs; Moro et al., 2010), nuocytes (Neill et al., 2010), innate helper type 2 (Ih2) cells (Price et al., 2010), or multipotent progenitors (MPPs; Saenz et al., 2010). Nuocytes and MPPs reside in mesenteric lymph nodes and spleen, NHCs are found in the fat-associated lymphoid tissue and Ih 2 cells are dispersed throughout the body, with most Ih 2 cells recovered from the liver. All studies in support of earlier work established an important role for ILC as IL-25 but also IL-33 dependent cells in the context of helminth infections. In a recent review those newly discovered cells were classified as innate helper cells type 2 (ILC2; Spits and Di Santo, 2011) compared with ROR $\gamma t$ expressing ILC subsets linked to cytokines such as IL-17 and IL-22 (ILC17 and ILC22). Lately, our own studies using an IL-9 fate reporter mouse model established that besides the Th2-type cytokines IL-5, IL-6, and IL-13 ILC2 also produce IL-9 (Wilhelm et al., 2011). However unlike IL-5 and IL-13 production in ILC, IL-9 is not permanently expressed but characterized by a very transient IL-2 dependent expression profile thus extending the portfolio of cytokines released by ILC.

\section{SURFACE MARKER EXPRESSION BY ILC}

Despite their obvious similarities all newly discovered ILC populations were given different names based on differential expression of surface markers. Interestingly, this variation is limited to surface markers indicative of the activation state of cells. For example CD25 and Sca-1 are upregulated on $T$ cells upon activation or differentiation. Thus, it is conceivable that the activation state or tissue distribution of the various innate lymphoid populations might influence the expression of surface receptors, as recently observed for nuocytes (Neill and Mckenzie, 2011). Indeed our own unpublished data suggests that ILC, which have expressed the IL-9 gene and are marked by eYFP expression $\left(\mathrm{eYFP}^{+}\right.$ILC), display variations of surface marker expression before and directly after papain challenge. Differences in the surface phenotype are also observed in ILC induced by intranasal delivery of either IL-25 or IL-33. While challenge with IL-33 induces ILC displaying an activated phenotype, with high expression of CD25 and Sca-1, IL-25 elicited ILC are low for those activation markers (our unpublished data). Interestingly, ILC generated by delivery of IL-33 are also more potent producers of IL-9 upon IL-2 stimulation, suggesting that their activation state could influence the ability to produce IL-9.

\section{DEPENDENCY ON THE ADAPTIVE IMMUNE SYSTEM (FIGURE 1)}

Another important but less explored finding is the observation that ILC may depend on the adaptive immune system for maintenance or expression of cytokines. This is best illustrated by the fact that nuocyte numbers (identified by IL-13 via GFP expression) in $\mathrm{Rag}^{-1-}$ mice are greatly reduced at day 6 but not at day 4 after Nippostrongylus brasiliensis infection in contrast to nuocytes induced in wild-type mice (Neill et al., 2010). There are two possible interpretations for this effect: Either nuocytes, while readily induced are not maintained in absence of the adaptive immune system or alternatively their characteristic cytokine expression is lost. Since the classification as nuocytes in this particular setting depended on continuous IL-13 expression monitored by GFP, loss of cytokine expression might erroneously be interpreted as loss of the whole population.

The latter assumption is supported by data from our laboratory in the context of papain mediated lung inflammation. Similar to the report from McKenzie et al. (1998) we observed reduced levels of IL-13 expression in $\mathrm{Rag}^{-/-}$mice in the absence of the adaptive immune system. Importantly though, we did not observe a significant loss of ILC in $\mathrm{Rag}^{-1-}$ mice based on the classification as lineage- ${ }^{-}$Thy $1.2^{+}$cells. Since IL-9 promotes expression of IL-5 and IL-13 from ILC and IL-2 induces IL-9, the lack of IL-2 from adaptive immune cells (or NKT cells), and the resulting loss of IL-9 expression is one possible explanation for reduced Th2type cytokine expression in $\mathrm{Rag}^{-/-}$mice. In line with this assumption, delivering either exogenous IL-2 or IL-9 to $\mathrm{Rag}^{-1-}$ mice in the context of allergen challenge resulted likewise in increased levels of IL-5 and IL-13 (unpublished data). However, delivery of 


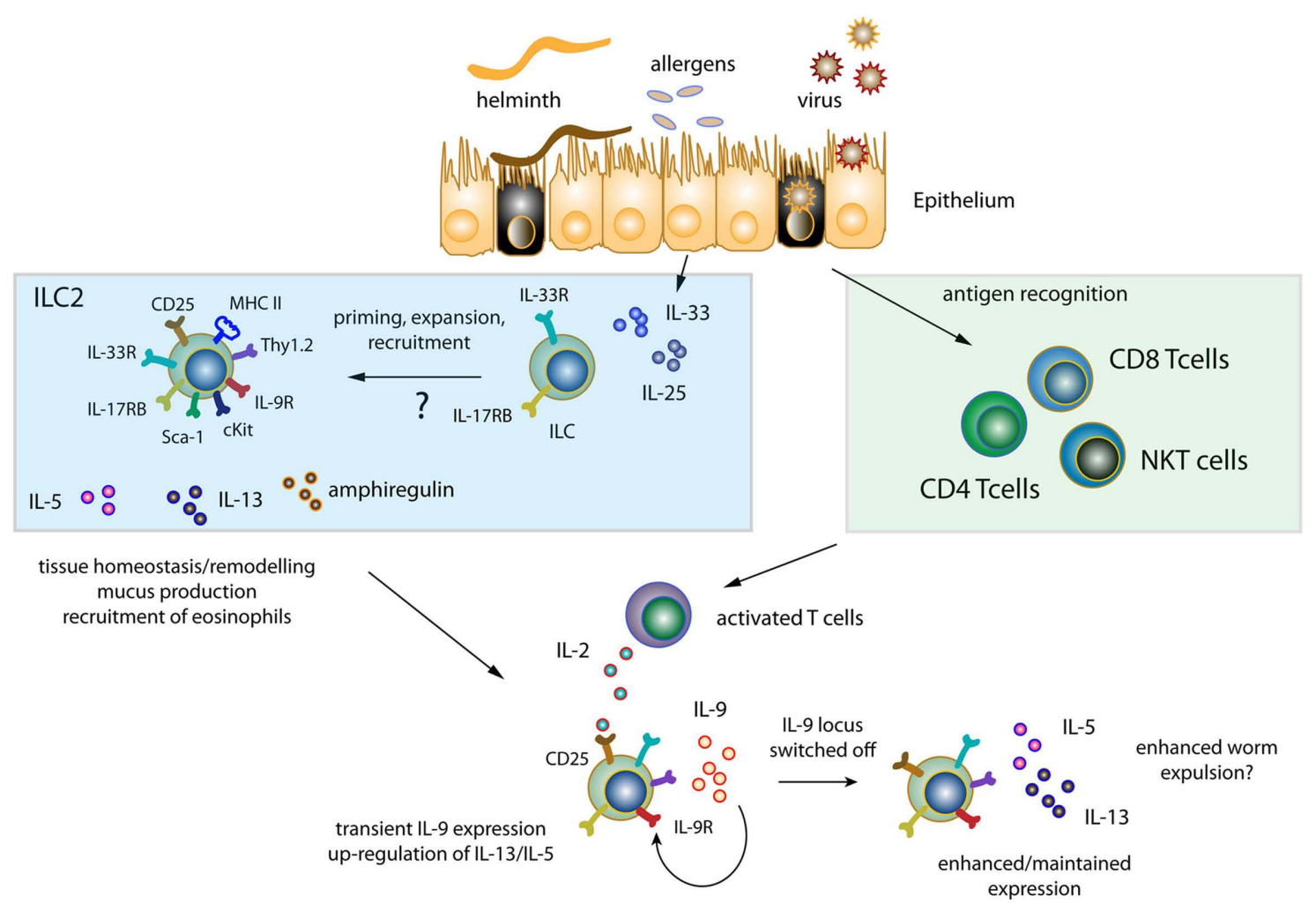

FIGURE 1 | Induction of innate lymphoid cell responses. Helminths, allergens, and viruses cause epithelial tissue damage resulting in the release of epithelial derived IL-33 and IL-25. Resident ILC populations are either activated and/or expand in response to IL-33 and IL-25 or are newly recruited to sites of inflammation. Primed/expanded/recruited ILC release IL-5, IL-13, and amphiregulin, while displaying variations of the surface molecules CD25, Thy 1.2, IL9R, cKit, Sca-1, IL17RB, and IL-33R dependent on their tissue distribution and activation status. Soluble factors released by ILC promote recruitment of eosinophils and features of airway hyperreactivity such as mucus production and tissue remodeling/repair. In addition, exposure to helminthes, viruses, and allergens results in antigen recognition and T cell activation (CD4, CD8, and NKT cells). T cell activation in turn leads to the release of IL-2, which induces transient IL-9 expression from ILC further enhancing/maintaining IL-5 and IL-13 expression thus possibly contributing to augmented worm expulsion.
IL-25 to $N$. brasiliensis infected $\mathrm{Rag}^{-1-}$ mice results in the rapid expulsion of worms, whereas such mice are normally highly susceptible to helminth infections (Price et al., 2010). Similar results are observed if $\mathrm{Rag}^{-1-} \mathrm{IL} 2 \mathrm{rg}^{-1-}$ (mice which lack ILC in addition to being deficient for T, B, and NK cells) are transferred with ILC and treated with IL-25 (Price et al., 2010). This suggests that continuous exposure to IL-25 and probably IL-33 is able to circumvent the dependency of the adaptive immune system and to substitute for IL-2 presence.

\section{WHAT IS THE RELATIVE CONTRIBUTION OF ILC TO THE OVERALL AMOUNT OF TYPE 2 CYTOKINES?}

The described dependency on the adaptive immune system either for cytokine expression or survival/maintenance is one of the most intriguing features of ILC. In the past, ablation of adaptive immune cells was believed to only affect adaptive sources of cytokine production. Since expression of cytokines from ILC depends on the adaptive immune system, it is conceivable that studies exploring the effect of $\mathrm{T}$ cell mediated cytokine expression unknowingly targeted cytokine expression from ILC simultaneously. In addition, antigen specific cytokine expression has been widely addressed by the restimulation of total splenocytes or lymphocytes with antigen in tissue culture. The presence of T cell (or NKT cell-) derived IL-2 in these cultures most likely will result in the induction of cytokine expression by ILC. This example clearly demonstrates that the cellular sources of cytokines have to be readdressed in the majority of experimental disease models.

It appears that the initiation of ILC happens around day 3-5 in an immune response, thus preceding the adaptive response (Neill et al., 2010; Price et al., 2010). However, whether ILC are needed for the induction of an adaptive type 2 immune response and/or whether they represent the critical and main source of cytokine producers during such immune responses is unknown. These questions are not trivial to address. While the initiation of the ILC response develops independent of the adaptive immune system, their maintenance requires adaptive immune cells. Here the study of $\mathrm{Rag}^{-/-}$mice can only deliver insufficient answers.

In a model of papain induced lung inflammation the majority of Th2-type cytokine producing cells originated from ILC despite the presence of fully developed Th2 cells (Wilhelm et al., 2011). Here we attempted to address the relative contribution to the overall amount of IL- 9 by transferring either $\mathrm{CD} 4^{+} \mathrm{T}$ cells or ILC to naïve 
$\mathrm{Rag}^{-/-}$IL2 $\mathrm{rg}^{-/-}$mice, thus limiting IL-9 production to either transferred $\mathrm{CD} 4^{+} \mathrm{T}$ cells or ILC. Despite the shortcomings of this experimental system, it clearly established that ILC are indeed the major producers of cytokines in this particular disease model. However, the question remains if we can relate these findings to other experimental systems and it needs to be addressed if type 2 cytokines produced during helminth infections, influenza or other disease models mainly originate from T cells or ILC. In line with the assumption that ILC are the major source of type 2 cytokines, an elegant set of experiments showed that the transfer of $\mathrm{CD}^{+} \mathrm{T}$ cells from il4/il1 $13^{-1-}$ mice to $\mathrm{Rag}^{-1-}$ mice, was sufficient to result in expulsion of $N$. brasiliensis from infected mice (Voehringer et al., 2006). Thus, the need for $\mathrm{T}$ cells was emphasized, but these could not have provided the critical source of IL-13, which is absolutely crucial for worm expulsion (Mckenzie et al., 1998; Urban et al., 1998). Further studies are needed to clearly establish the link between the adaptive immune system and the regulation of ILC in different experimental models.

\section{THE FUNCTION OF ILC IN INFLUENZA INFECTION}

Two recent studies explored the function of lung resident ILC in the context of influenza infection (Chang et al., 2011, p. 103; Monticelli, 2011, p 170). The first study explored the function of innate lymphocytes during the early phase of influenza infection and established that obstruction of the airways during flu infections seems to be dependent on IL-13 by ILC. Interestingly, a second study that focused on the late phase after infection suggested an important role for ILC in the maintenance of airway epithelial integrity, showing that ablation of ILC in $\mathrm{Rag}^{-/-}$mice during the repair phase after influenza mediated lung infection leads to diminished lung function and impaired airway remodeling in mice. This process appeared to be independent of IL-13. However, administration of amphiregulin was able to restore airway epithelial integrity and tissue homeostasis, thus suggesting a previously unrecognized function of ILC via the expression of amphiregulin. The involvement - if any - of IL-9 in these processes remains to be addressed.

\section{ILC RESPONSES IN HUMANS}

The first evidence for the presence of ILC in humans showed that NHC similar to those of the mouse are present in human fatassociated lymphoid clusters (FALC; Moro et al., 2010). Another, more recent study demonstrated that nasal polyps isolated from patients with atopic disease contain increased numbers of ILC (Mjosberg et al., 2011). These cells were characterized by the expression of the chemoattractant receptor CRTH2 (chemoattractant receptor-homologous molecule expressed on Th2 lymphocytes), were present in lung, gut, and nasal tissues and produced IL-13 in response to stimulation with combinations of IL-2 and IL-25 or IL-33. This strongly suggests that similar mechanism for the induction of ILC in mouse and human exists and highlights their potential in atopic diseases like asthma and allergies.

\section{FUTURE DIRECTIONS}

While the accumulation of ILC has been shown to depend on IL-25 and/or IL-33, it is not entirely clear if IL-25 and IL-33 exposure lead to their recruitment or expansion. In vitro studies suggest that combinations of IL-33 and IL-2 or IL-7 are able to expand the pool of existing ILC. However it remains unknown if ILC are recruited from other sites of the body like liver or bone marrow during an ongoing immune response. Additionally, no data is available with regard to which progenitor cells give rise to ILC.

Finally, the evolutionary origin of ILC remains an interesting as yet unsolved question. Did ILC appear before the development of the adaptive immune system, or did ILC and the adaptive immune system co-evolve as their dependency on the adaptive immune system might suggest? These questions will be hopefully addressed in the future to further help to shape our understanding of this intriguing subset of innate lymphocytes.

\section{CONCLUDING REMARIKS ABOUT THE PHYSIOLOGICAL ROLE OF ILC IN GENERAL}

It appears that ILC play an important role not only in the initiation and effector phase of an immune response but also in the resolution of inflammation and the tissue repair process. Most of these functions are also attributed to $\mathrm{T}$ cells and $\mathrm{T}$ cell derived cytokines. However, it seems unlikely that such ILC functions have just evolved as mirror images of those in adaptive T cells. A consideration is the potential of adaptive $\mathrm{T}$ cell responses to cause immune pathology or autoimmune syndromes. Although ILC responses have in some cases been shown to have detrimental effects (Buonocore et al., 2010; Chang et al., 2011) these studies by necessity had to be done in relatively contrived experimental models with immunocompromised mice, thus not necessarily reflecting similar pathogenic actions of ILC in wild-type mice. It therefore remains a possibility that immune responses by ILC are a first line of defense coupled with regenerative potential, whereas exacerbation and disbalance toward pathology would only be encountered following the involvement of adaptive T cells. To test any such predictions it would be necessary to develop experimental systems that would allow the inducible deletion of just ILC rather than the current models that can only study ILC function in the absence of an adaptive immune system.

\section{REFERENCES}

Buonocore, S., Ahern, P. P., Uhlig, H. H., Ivanov, I. I., Littman, D. R., Maloy, K. J., and Powrie, F. (2010). Innate lymphoid cells drive interleukin-23-dependent innate intestinal pathology. Nature 464, 1371-1375.

Chang, Y. J., Kim, H. Y., Albacker, L. A., Baumgarth, N., Mckenzie, A. N., Smith, D. E., Dekruyff, R. H., and Umetsu, D. T. (2011). Innate lymphoid cells mediate influenza-induced airway hyper-reactivity independently of adaptiveimmunity. Nat.Immunol.12,631-638.

Fallon, P. G., Ballantyne, S. J., Mangan, N. E., Barlow, J. L., Dasvarma, A., Hewett, D. R., Mcilgorm, A., Jolin, H. E., and Mckenzie, A. N. (2006). Identification of an interleukin (IL)-25-dependent cell population that provides IL-4, IL-5, and IL-13 at the onset of helminth expulsion. J. Exp. Med. 203, 1105-1116.

Fort, M. M., Cheung, J., Yen, D., Li, J., Zurawski, S. M., Lo, S., Menon, S., Clifford, T., Hunte, B., Lesley, R., Muchamuel, T., Hurst, S. D., Zurawski, G., Leach, M. W., Gorman, D. M., and Rennick, D. M. (2001).IL-25 induces IL-4, IL-5, and IL-13 and Th2-associated pathologies in vivo. Immunity 15, 985-995.

Hurst, S. D., Muchamuel, T., Gorman, D. M., Gilbert, J. M., Clifford, T., Kwan, S., Menon, S., Seymour, B., Jackson, C., Kung, T. T., Brieland, J. K., Zurawski, S. M., Chapman, R. W., Zurawski, G., and Coffman, R. L. (2002). New IL-17 family members promote Th1 or Th2 responses in the lung: in vivo function of the novel cytokine IL-25. J. Immunol. 169, 443-453.

Mckenzie, G. J., Bancroft, A., Grencis, R. K., and Mckenzie, A. N. (1998). A distinct role for interleukin-13 in Th2-cell-mediated immune responses. Curr. Biol. 8, 339-342.

Mjosberg, J. M., Trifari, S., Crellin, N. K., Peters, C. P., Van Drunen, C. M., Piet, B., Fokkens, W. J., Cupedo, T., and Spits, H. (2011). Human IL-25- and IL-33- 
responsive type 2 innate lymphoid cells are defined by expression of CRTH2 and CD161. Nat. Immunol. 12, 1055-1062.

Monticelli, L.A., Sonnenberg, G. F., Abt, M. C., Alenghat, T., Ziegler, C. G., Doering, T. A., Angelosanto, J. M., Laidlaw, B. J., Yang, C. Y., Sathaliyawala, T., Kubota, M., Turner, D., Diamond, J.M., Goldrath,A. W., Farber, D. L., Collman, R. G., Wherry, E. J., and Artis, D. (2011). Innate lymphoid cells promote lung-tissue homeostasis after infection with influenza virus. Nature Immunol. doi: 10.1031/ni.2131

Moro, K., Yamada, T., Tanabe, M., Takeuchi, T., Ikawa, T., Kawamoto, H., Furusawa, J., Ohtani, M., Fujii, H., and Koyasu, S. (2010). Innate production of $\mathrm{T}(\mathrm{H}) 2$ cytokines by adipose tissue-associated c-Kit(+)Sca-1(+) lymphoid cells. Nature 463, 540-544.

Neill, D. R., and Mckenzie, A. N. (2011). Nuocytes and beyond: new insights into helminth expulsion. Trends Parasitol. 27, 214-221.

Neill, D. R., Wong, S. H., Bellosi, A., Flynn, R. J., Daly, M., Langford, T. K., Bucks, C., Kane, C. M., Fallon, P. G., Pannell, R., Jolin, H. E., and Mckenzie, A. N. (2010). Nuocytes represent a new innate effector leukocyte that mediates type- 2 immunity. Nature 464, 1367-1370.
Paul, W. E., and Zhu, J. F. (2010). How are T(H)2-type immune responses initiated and amplified? Nat. Rev. Immunol. 10, 225-235.

Price, A. E., Liang, H. E., Sullivan, B. M., Reinhardt, R. L., Eisley, C. J., Erle, D. J., and Locksley, R. M. (2010). Systemically dispersed innate IL-13-expressing cells in type 2 immunity. Proc. Natl. Acad. Sci. U.S.A. 107, 11489-11494.

Saenz, S. A., Siracusa, M. C., Perrigoue, J. G., Spencer, S. P., Urban, J. F. Jr., Tocker, J. E., Budelsky, A. L., Kleinschek, M.A., Kastelein, R. A., Kambayashi, T., Bhandoola, A. and Artis, D. (2010). IL25 elicits a multipotent progenitor cell population that promotes $\mathrm{T}(\mathrm{H}) 2$ cytokine responses. Nature 464, 1362-1366.

Sawa, S., Cherrier, M., Lochner, M., Satoh-Takayama, N., Fehling, H. J., Langa, F., Di Santo, J. P., and Eberl, G. (2010). Lineage relationship analysis of RORgammat+ innate lymphoid cells. Science 330, 665-669.

Spits, H., and Di Santo, J. P. (2011). The expanding family of innate lymphoid cells: regulators and effectors of immunity and tissue remodeling. Nat. Immunol. $12,21-27$.

Urban, J. F. Jr., Noben-Trauth, N., Donaldson, D D., Madden, K. B., Morris, S. C., Collins, M., and Finkelman, F. D. (1998). IL-13, IL-4Ralpha, and Stat6 are required for the expulsion of the gastrointesti- nal nematode parasite Nippostrongylus brasiliensis. Immunity 8, 255-264.

Voehringer, D., Reese, T. A., Huang, X., Shinkai, K., and Locksley, R. M. (2006). Type 2 immunity is controlled by IL-4/IL-13 expression in hematopoietic non-eosinophil cells of the innate immune system. J. Exp. Med. 203, 1435-1446.

Wilhelm, C., Hirota, K., Stieglitz, B., Van Snick, J. Tolaini, M., Lahl, K., Sparwasser, T., Helmby, H., and Stockinger, B. (2011). An IL-9 fate reporter demonstrates the induction of an innate IL-9 response in lung inflammation. Nat. Immunol. 12, 1071-1077.

Received: 04 November 2011; accepted: 12 November 2011; published online: 28 November 2011.

Citation: Wilhelm C and Stockinger B (2011) Innate lymphoid cells and type 2 (Th2) mediated immune responses pathogenic or beneficial? Front. Immun. 2:68. doi: 10.3389/ fimmu.2011.00068

This article was submitted to Frontiers in Inflammation, a specialty of Frontiers in Immunology.

Copyright (@) 2011 Wilhelm and Stockinger. This is an openaccess article distributed under the terms of the Creative Commons Attribution Non Commercial License, which permits use, distribution, and reproduction in other forums, provided the original authors and source are credited. 